\title{
Comparison of radiation hazard of HLW in several spent nuclear fuel reprocessing scenarios
}

\author{
A. Ochkin ${ }^{1}$, D. Gladilov ${ }^{1}$ and S. Stefanovsky ${ }^{2}$ \\ ${ }^{1}$ D.Mendeleev University of Chemical Technology of Russia, Moscow, Russia \\ ${ }^{2}$ SIA «RADON», Moscow, Russia
}

\begin{abstract}
Radiation hazard of radionuclide has been calculated as a product of $A \varepsilon$ where $A$ is an activity of radionuclide and $\varepsilon$ is a dose coefficient through ingestion. The values $A \varepsilon$ of 18 radionuclide in spent fuel of WWER-440 are calculated. Because the full division of americium and curium from HLW is very complicated a separation americium from curium is considered. It is shown that a separation of americium in a special fraction allows decreasing the radiation hazard of HLW by $97.6 \%$ after 1000 years.
\end{abstract}

\section{Introduction}

The modern fuel reprocessing is based on PUREX-process. Resulting HLW contains less than $0.01 \%$ of uranium, $0.025 \%$ of plutonium and $0.5 \%$ of neptunium [1]. Other radionuclides remaining in HLW are: americium, curium and fission products. Today's reality requires more safety. It is necessary to decrease contents of americium and curium in HLW making it significantly safer for future storage [2]. Separation of only americium can be considered as a partial measure due to significant impact of curium on radiation hazard in period around 700-800 years [2]. The aim of this work is comparison of radiation safety in those scenarios of HLW management.

\section{Experimental data}

Composition of radionuclide in spent fuel of WWER-440 [3] is given in Table 1. This data was taken as a base. Then activities of radionuclide are calculated for three periods: after stop, after 5 years storage and after reprocessing and dividing of uranium, plutonium and neptunium as in [1].

Table 1. Composition of spent fuel of WWER-440 [3] and activities of actinides

\begin{tabular}{|c|c|c|c|c|c|c|}
\hline \multirow{2}{*}{$\begin{array}{l}\text { Radio- } \\
\text { nuclide }\end{array}$} & \multirow{2}{*}{$\begin{array}{l}\mathrm{T}_{1 / 2} \\
\text { years }\end{array}$} & \multirow{2}{*}{$\begin{array}{c}\text { Dose } \\
\text { coeffici- } \\
\text { ent } \varepsilon \cdot 10^{7} \text {, } \\
\mathrm{Sv} / \mathrm{Bq}\end{array}$} & \multirow{2}{*}{$\begin{array}{c}\text { Content, } \\
\mathrm{kg} / \mathrm{t} \mathrm{U} \\
{[3]}\end{array}$} & \multicolumn{3}{|c|}{ Activity $A, \mathrm{~Bq} / \mathrm{t} \mathrm{U}$} \\
\hline & & & & $\begin{array}{l}\text { After } \\
\text { stop }\end{array}$ & $\begin{array}{c}\text { After } 5 \\
\text { years } \\
\text { storage }\end{array}$ & $\begin{array}{c}\text { After } \\
\text { repro- } \\
\text { cessing }\end{array}$ \\
\hline 1 & 2 & 3 & 4 & 5 & 6 & 7 \\
\hline${ }^{232} \mathrm{U}$ & 68.9 & 3.3 & $9.00 \cdot 10^{-1}$ & $7.44 \cdot 10^{8}$ & $1.59 \cdot 10^{9}$ & $1.59 \cdot 10^{5}$ \\
\hline${ }^{234} \mathrm{U}$ & $2.45 \cdot 10^{5}$ & 0.49 & 0.215 & $4.96 \cdot 10^{10}$ & $5.09 \cdot 10^{10}$ & $5.09 \cdot 10^{6}$ \\
\hline${ }^{235} \mathrm{U}$ & $7.04 \cdot 10^{8}$ & 0.47 & 11.2 & $8.95 \cdot 10^{8}$ & $8.96 \cdot 10^{8}$ & $8.96 \cdot 10^{4}$ \\
\hline${ }^{236} \mathrm{U}$ & $2.34 \cdot 10^{\prime}$ & 0.47 & 4.50 & $1.08 \cdot 10^{10}$ & $1.08 \cdot 10^{I I}$ & $1.08 \cdot 10^{\prime}$ \\
\hline${ }^{238} \mathrm{U}$ & $4.47 \cdot 10^{9}$ & 0.45 & 941 & $1.17 \cdot 10^{10}$ & $1.17 \cdot 10^{10}$ & $1.17 \cdot 10^{6}$ \\
\hline${ }^{231} \mathrm{~Np}$ & $2.14 \cdot 10^{6}$ & 1.1 & 0.445 & $1.16 \cdot 10^{10}$ & $4.71 \cdot 10^{9}$ & $2.35 \cdot 10^{7}$ \\
\hline${ }^{236} \mathrm{Pu}$ & 2.85 & 2.0 & $1.60 \cdot 10^{-6}$ & $3.15 \cdot 10^{10}$ & $9.41 \cdot 10^{9}$ & $2.35 \cdot 10^{6}$ \\
\hline${ }^{238} \mathrm{Pu}$ & 87.7 & 2.3 & 0.137 & $8.67 \cdot 10^{13}$ & $9.16 \cdot 10^{13}$ & $2.29 \cdot 10^{10}$ \\
\hline
\end{tabular}




\begin{tabular}{|c|c|c|c|c|c|c|}
\hline 1 & 2 & 3 & 4 & 5 & 6 & 7 \\
\hline${ }^{239} \mathrm{Pu}$ & $2.41 \cdot 10^{4}$ & 2.5 & 5.37 & $1.23 \cdot 10^{13}$ & $1.23 \cdot 10^{13}$ & $3.08 \cdot 10^{9}$ \\
\hline${ }^{240} \mathrm{Pu}$ & $6.56 \cdot 10^{3}$ & 2.5 & 2.17 & $1.82 \cdot 10^{13}$ & $1.82 \cdot 10^{13}$ & $4.56 \cdot 10^{9}$ \\
\hline${ }^{241} \mathrm{Pu}$ & 14.4 & 0.048 & 1.06 & $4.04 \cdot 10^{15}$ & $3.18 \cdot 10^{15}$ & $7.94 \cdot 10^{11}$ \\
\hline${ }^{242} \mathrm{Pu}$ & $3.76 \cdot 10^{5}$ & 2.4 & 0.430 & $6.25 \cdot 10^{10}$ & $6.25 \cdot 10^{10}$ & $1.56 \cdot 10^{7}$ \\
\hline${ }^{241} \mathrm{Am}$ & 432 & 2.0 & 0.0410 & $5.20 \cdot 10^{12}$ & $3.38 \cdot 10^{13}$ & $3.38 \cdot 10^{13}$ \\
\hline${ }^{242 \mathrm{~m}} \mathrm{Am}$ & 152 & 1.9 & $6.40 \cdot 10^{-4}$ & $2.30 \cdot 10^{11}$ & $2.25 \cdot 10^{11}$ & $2.25 \cdot 10^{11}$ \\
\hline${ }^{243} \mathrm{Am}$ & $7.38 \cdot 10^{3}$ & 2.0 & 0.0490 & $3.61 \cdot 10^{11}$ & $3.61 \cdot 10^{11}$ & $3.61 \cdot 10^{11}$ \\
\hline${ }^{242} \mathrm{Cm}$ & 0.446 & 0.12 & 0.0136 & $1.67 \cdot 10^{15}$ & $9.29 \cdot 10^{11}$ & $9.29 \cdot 10^{11}$ \\
\hline${ }^{243} \mathrm{Cm}$ & 28.5 & 1.5 & $3.80 \cdot 10^{-4}$ & $7.26 \cdot 10^{11}$ & $6.45 \cdot 10^{11}$ & $6.45 \cdot 10^{11}$ \\
\hline${ }^{244} \mathrm{Cm}$ & 18.1 & 1.2 & 0.0197 & $5.89 \cdot 10^{13}$ & $4.87 \cdot 10^{13}$ & $4.87 \cdot 10^{13}$ \\
\hline${ }^{245} \mathrm{Cm}$ & $8.50 \cdot 10^{3}$ & 2.1 & $1.03 \cdot 10^{-3}$ & $6.54 \cdot 10^{9}$ & $6.54 \cdot 10^{9}$ & $6.54 \cdot 10^{9}$ \\
\hline${ }^{246} \mathrm{Cm}$ & $4.73 \cdot 10^{3}$ & 2.1 & $6.90 \cdot 10^{-5}$ & $7.84 \cdot 10^{5}$ & $7.84 \cdot 10^{5}$ & $7.84 \cdot 10^{5}$ \\
\hline & & $\sum$ & $\mathbf{9 6 7}$ & $\mathbf{5 . 8 9} \cdot \mathbf{1 0}^{15}$ & $\mathbf{3 . 3 8} \cdot \mathbf{1 0}^{15}$ & $\mathbf{8 . 5 7 \cdot 1 0}$ \\
\hline
\end{tabular}

After 5 years storage activities of some radionuclide became less and it was seen from Table 2 .

Table 2. Decay of some radionuclide after 5 years storage.

\begin{tabular}{|c|c|c|c|c|c|c|}
\hline Radio- & $\mathrm{T}_{1 / 2}$, & \multicolumn{2}{|c|}{ Content $\mathrm{g} / \mathrm{t} \mathrm{U}$} & \multicolumn{3}{|c|}{ Decay products } \\
\cline { 3 - 7 } nuclide & year & Initial & Final & Nuclide & Mass g/t U & $\mathrm{Bq} / \mathrm{t} \mathrm{U}$ \\
\hline${ }^{241} \mathrm{Pu}$ & 14.4 & 1060 & 832 & ${ }^{241} \mathrm{Am}$ & 228 & $2.86 \cdot 10^{13}$ \\
\hline${ }^{242} \mathrm{Cm}$ & 0.446 & 13.6 & 0.006 & ${ }^{238} \mathrm{Pu}$ & 13.5 & $8.49 \cdot 10^{12}$ \\
\hline${ }^{243} \mathrm{Cm}$ & 28.5 & 0.38 & 0.34 & ${ }^{239} \mathrm{Pu}$ & 0.04 & $0.8 \cdot 10^{8}$ \\
\hline${ }^{244} \mathrm{Cm}$ & 18.1 & 19.7 & 16.3 & ${ }^{240} \mathrm{Pu}$ & 3.4 & $2.87 \cdot 10^{10}$ \\
\hline
\end{tabular}

\section{Radiation hazard}

The activities of original radionuclide together with their decay products were calculated as function of time. The radiation hazard of radionuclide is calculated as a product of $A \varepsilon$ where $A$ is an activity of radionuclide and $\varepsilon$ is a dose coefficient through ingestion [4]. The results of the calculation are given in Table 3 and they only include dose coefficients of radionuclide with $T_{1 / 2}>15$ days. There are 18 nuclide presented in Table 1 after reprocessing (column 7). The data in Table 3 (column 2) presented $A \varepsilon$ at 0 . The data at other columns were calculated from the data at column 2 and decay results for column 2. Therefore it is possible to divide the data $A \varepsilon$ into 18 lines each corresponding to the radiation hazard in various time periods.

Table 3. Alteration of radiation hazard of actinides during HLW storage

\begin{tabular}{|c|c|c|c|c|c|c|c|}
\hline \multirow{2}{*}{$\begin{array}{c}\text { Radio- } \\
\text { nuclide }\end{array}$} & \multicolumn{7}{|c|}{ Radiation hazard $A \cdot \varepsilon$, Sv $\mathrm{U}$ after storage during } \\
\cline { 2 - 8 } & 0 years & 10 years & $\begin{array}{c}1000 \\
\text { years }\end{array}$ & $\begin{array}{c}10000 \\
\text { years }\end{array}$ & $\begin{array}{c}50000 \\
\text { years }\end{array}$ & $\begin{array}{c}10^{5} \\
\text { years }\end{array}$ & $\begin{array}{c}10^{6} \\
\text { years }\end{array}$ \\
\hline 1 & 2 & 3 & 4 & 5 & 6 & 7 & 8 \\
\hline${ }^{232} \mathrm{U}$ & $5.23 \cdot 10^{-2}$ & 0.0577 & $0^{*}$ & 0 & 0 & 0 & 0 \\
\hline${ }^{234} \mathrm{U}$ & 0.249 & 0.249 & 0.276 & 1.07 & 4.18 & 6.27 & 1.06 \\
\hline${ }^{235} \mathrm{U}$ & $4.21 \cdot 10^{-3}$ & $4.23 \cdot 10^{-3}$ & $7.73 \cdot 10^{-3}$ & 0.0369 & 0.116 & 0.155 & 0.176 \\
\hline${ }^{236} \mathrm{U}$ & $5.06 \cdot 10^{-2}$ & 0.0506 & 0.0506 & 0.0506 & 0.0505 & 0.0505 & 0.0492 \\
\hline${ }^{238} \mathrm{U}$ & $5.27 \cdot 10^{-2}$ & 0.0566 & 0.0568 & 0.0605 & 0.132 & 0.304 & 2.62 \\
\hline${ }^{235} \mathrm{~Np}$ & 6.4 & 6.45 & 6.47 & 7.07 & 12.3 & 18.1 & 33.3 \\
\hline${ }^{236} \mathrm{Pu}$ & 0.47 & 0.0743 & 0 & 0 & 0 & 0 & 0 \\
\hline${ }^{238} \mathrm{Pu}$ & $5.27 \cdot 10^{3}$ & $5.27 \cdot 10^{3}$ & 2.38 & 1.69 & 6.66 & 10.0 & 16.9 \\
\hline${ }^{239} \mathrm{Pu}$ & 770 & 770 & 749 & 578 & 183 & 43.6 & 0.207 \\
\hline${ }^{240} \mathrm{Pu}$ & $1.14 \cdot 10^{3}$ & $1.14 \cdot 10^{3}$ & $1.03 \cdot 10^{3}$ & 39.6 & 5.85 & 0.0893 & 0.0584 \\
\hline${ }^{24} \mathrm{Pu}$ & $3.81 \cdot 10^{3}$ & $4.36 \cdot 10^{3}$ & $1.10 \cdot 10^{3}$ & 0.644 & 1.12 & 1.66 & 3.06 \\
\hline${ }^{242} \mathrm{Pu}$ & 3.75 & 3.75 & 3.74 & 3.68 & 3.42 & 3.12 & 0.592 \\
\hline 1 & 2 & 3 & 4 & 5 & 6 & 7 & 8 \\
\hline$(\mathbf{1}) \sum \mathbf{U}+\mathbf{P u}$ & $\mathbf{1 . 1 0 \cdot 1 0 ^ { 4 }}$ & $\mathbf{1 . 1 1 \cdot 1 0 ^ { 4 }}$ & $\mathbf{2 . 8 9} \cdot \mathbf{1 0}$ & $\mathbf{6 3 1}$ & $\mathbf{2 1 7}$ & $\mathbf{8 3 . 3}$ & $\mathbf{5 8 . 0}$ \\
\hline
\end{tabular}




\begin{tabular}{|c|c|c|c|c|c|c|c|}
\hline${ }^{242} \mathrm{Cm}$ & $1.11 \cdot 10^{4}$ & $1.00 \cdot 10^{3}$ & 0.490 & 0.346 & 1.37 & 2.05 & 0.348 \\
\hline${ }^{243} \mathrm{Cm}$ & $9.66 \cdot 10^{4}$ & $7.62 \cdot 10^{4}$ & 189 & 146 & 46.2 & 11.0 & 0.0523 \\
\hline${ }^{244} \mathrm{Cm}$ & $5.84 \cdot 10^{6}$ & $3.99 \cdot 10^{6}$ & $3.03 \cdot 10^{4}$ & $1.17 \cdot 10^{4}$ & 173 & 2.63 & 1.72 \\
\hline${ }^{245} \mathrm{Cm}$ & $1.37 \cdot 10^{3}$ & $1.39 \cdot 10^{3}$ & $2.28 \cdot 10^{3}$ & $1.23 \cdot 10^{3}$ & 51.9 & 8.26 & 14.9 \\
\hline${ }^{246} \mathrm{Cm}$ & 0.165 & 0.164 & 0.143 & 0.0402 & 0.0023 & 0.0020 & $0^{*}$ \\
\hline (2) $\sum \mathrm{Cm}$ & $5.95 \cdot 10^{6}$ & $4.08 \cdot 10^{6}$ & $3.57 \cdot 10^{4}$ & $1.31 \cdot 10^{4}$ & 272 & 23.9 & 17.0 \\
\hline${ }^{241} \mathrm{Am}$ & $6.76 \cdot 10^{6}$ & $6.65 \cdot 10^{6}$ & $1.36 \cdot 10^{6}$ & 824 & $1.43 \cdot 10^{3}$ & $2.12 \cdot 10^{3}$ & $3.91 \cdot 10^{3}$ \\
\hline${ }^{242 \mathrm{~m}} \mathrm{Am}$ & $4.26 \cdot 10^{4}$ & $4.68 \cdot 10^{4}$ & $1.29 \cdot 10^{3}$ & 26.1 & 105 & 158 & 26.7 \\
\hline${ }^{243} \mathrm{Am}$ & $7.22 \cdot 10^{4}$ & $7.22 \cdot 10^{4}$ & $6.82 \cdot 10^{4}$ & $4.25 \cdot 10^{4}$ & $9.74 \cdot 10^{3}$ & $2.25 \cdot 10^{3}$ & 7.43 \\
\hline (3) $\sum$ Am & $6.87 \cdot 10^{6}$ & $1.09 \cdot 10^{7}$ & $1.47 \cdot 10^{6}$ & $4.34 \cdot 10^{4}$ & $1.13 \cdot 10^{4}$ & $4.53 \cdot 10^{3}$ & $3.94 \cdot 10^{3}$ \\
\hline
\end{tabular}

Table 4. The data for some fission products.

\begin{tabular}{|c|c|c|c|c|c|c|c|c|}
\hline \multirow{2}{*}{$\begin{array}{l}\text { Rardio- } \\
\text { nuclide }\end{array}$} & \multirow{2}{*}{$\begin{array}{l}T_{1 / 2}, \\
\text { years }\end{array}$} & \multirow{2}{*}{$\begin{array}{c}\text { Yield at } \\
{ }^{235} \mathrm{U}^{239} \mathrm{Pu}, \%\end{array}$} & \multirow{2}{*}{$\begin{array}{c}\text { Dose } \\
\text { coeffi- } \\
\text { cient } \\
\varepsilon \cdot 10^{7}, \\
\mathrm{~Sv} / \mathrm{Bq}\end{array}$} & \multirow{2}{*}{$\begin{array}{c}\text { Activity } \\
A_{0}, \\
\mathrm{~Bq} / \mathrm{t}\end{array}$} & \multicolumn{4}{|c|}{ Radiation hazard $A \cdot \varepsilon, \mathrm{Sv} / \mathrm{t} \mathrm{U}$} \\
\hline & & & & & $\begin{array}{c}0 \\
\text { years }\end{array}$ & $\begin{array}{l}500 \\
\text { years }\end{array}$ & $\begin{array}{l}1000 \\
\text { years }\end{array}$ & $\begin{array}{c}50000 \\
\text { years }\end{array}$ \\
\hline${ }^{79} \mathrm{Se}$ & $6.5 \cdot 10^{4}$ & $\begin{array}{c}0.0453 / \\
0.0470\end{array}$ & 2.9 & $1.4 \cdot 10^{10}$ & 28.0 & 28.0 & 28.0 & 26.0 \\
\hline${ }^{90} \mathrm{Sr} /{ }^{90} \mathrm{Y}$ & 29.1 & $5.90 / 2.10$ & $28+2.7$ & $2.73 \cdot 10^{15}$ & $9.2 \cdot 10^{7}$ & 621 & 0 & 0 \\
\hline${ }^{95} \mathrm{Zr}$ & $1.53 \cdot 10^{6}$ & $6.39 / 3.90$ & 1.1 & $6.7 \cdot 10^{10}$ & 88.0 & 88.0 & 88.0 & 86.0 \\
\hline${ }^{99} \mathrm{Tc}$ & $2.13 \cdot 10^{5}$ & $6.11 / 6.14$ & 0.64 & $5.7 \cdot 10^{11}$ & 375 & 375 & 375 & 318 \\
\hline${ }^{10 /} \mathrm{Pd}$ & $6.5 \cdot 10^{6}$ & $0.139 / 3.36$ & 0.037 & $5.3 \cdot 10^{9}$ & 0.0295 & 0.0295 & 0.0295 & 0.0293 \\
\hline${ }^{126} \mathrm{Sn}$ & $1.0 \cdot 10^{5}$ & $0.055 / 0.266$ & 4.7 & $3.2 \cdot 10^{10}$ & 33.4 & 33.4 & 33.4 & 28.8 \\
\hline${ }^{129} \mathrm{I}$ & $1.57 \cdot 10^{\prime}$ & $0.353 / 0.749$ & 110 & $7.0 \cdot 10^{8}$ & 92.0 & 92.0 & 92.0 & 91.8 \\
\hline${ }^{139} \mathrm{Cs}$ & $2.3 \cdot 10^{6}$ & $6.53 / 7.62$ & 2.0 & $1.50 \cdot 10^{10}$ & 30.0 & 30.0 & 30.0 & 29.6 \\
\hline${ }^{13 /} \mathrm{Cs}$ & 30.0 & $6.27 / 6.73$ & 13 & $4.30 \cdot 10^{15}$ & $5.2 \cdot 10^{\prime}$ & 515 & 0 & 0 \\
\hline
\end{tabular}

\section{Possible scenarios of fuel reprocessing}

Then radionuclide from Table 3 can be divided in three groups. The first group (I) included the radionuclide of uranium, neptunium and plutonium. The second group (II) included the radionuclide of curium and the thirst group (III) included the radionuclide of americium. Besides data for some important fission products are included in Table 4. The data $A \varepsilon$ for fission products is decreased fast from 0 to $500 \div 1000$ years due to decay of strontion-90 and cesium-137. The data $A \varepsilon$ for fission products after 1000 years is lightly more than $A \varepsilon$ for actinides.

Table 5. Possible scenarios of fuel reprocessing.

\begin{tabular}{|c|c|c|c|c|c|c|}
\hline \multirow{2}{*}{$\begin{array}{c}\text { Radio- } \\
\text { nuclide }\end{array}$} & \multicolumn{5}{|c|}{ Radiation hazard $A \cdot \varepsilon, \mathrm{Sv} / \mathrm{U}$ after storage during } \\
\cline { 2 - 7 } & 0 years & 10 years & $\begin{array}{c}1000 \\
\text { years }\end{array}$ & $\begin{array}{c}50000 \\
\text { years }\end{array}$ & $\begin{array}{c}10^{5} \\
\text { years }\end{array}$ & $\begin{array}{c}10^{6} \\
\text { years }\end{array}$ \\
\hline Scenario I. $\sum(\mathrm{U}+\mathrm{Np}+\mathrm{Pu})$ & 11000 & 11100 & 2890 & 217 & 83.3 & 58.0 \\
\hline$\sum \mathrm{Cm}$ & $5.95 \cdot 10^{6}$ & $4.07 \cdot 10^{6}$ & $3.28 \cdot 10^{4}$ & 272 & 23.9 & 17.0 \\
\hline Scenario II. $\sum(\mathrm{U}+\mathrm{Np}+\mathrm{Pu}+\mathrm{Cm})$ & $5.96 \cdot 10^{6}$ & $4.08 \cdot 10^{6}$ & $3.57 \cdot 10^{4}$ & 489 & 107 & 75.0 \\
\hline$\sum \mathrm{Am}$ & $6.87 \cdot 10^{6}$ & $6.77 \cdot 10^{6}$ & $1.43 \cdot 10^{6}$ & 11300 & 4530 & 3940 \\
\hline Scenario III. $\sum(\mathrm{U}+\mathrm{Np}+\mathrm{Pu}+\mathrm{Cm}+\mathrm{Am})$ & $1.28 \cdot 10^{7}$ & $1.08 \cdot 10^{7}$ & $1.47 \cdot 10^{6}$ & 11800 & 4640 & 4020 \\
\hline$\sum \mathrm{Am} / \sum(\mathrm{U}+\mathrm{Np}+\mathrm{Pu}+\mathrm{Cm}+\mathrm{Am})$ & 0.536 & 0.624 & 0.976 & 0.958 & 0.977 & 0.981 \\
\hline Fission products & $14.0 \cdot 10^{7}$ & - & 646 & 580 & - & - \\
\hline
\end{tabular}

The data Table 5 can be used to divide three possible scenarios:

Scenario I. Americium and curium are separated in other residue. HLW contain uranium, plutonium and neptunium. Concentrations of americium and curium are known. This scenario can be considered as ideal. 
Scenario II. Americium is separated solely while curium remains in HLW [2]. The effect of americium separation was calculated as relation of $\sum \mathrm{Am} / \sum(\mathrm{U}+\mathrm{Np}+\mathrm{Pu}+\mathrm{Cm}+\mathrm{Am})$.

Scenario III. The values of $A \varepsilon$ are maximal as americium and curium together with uranium, neptunium and plutonium are all part of nuclear waste. This process is similar to currently used.

\section{Conclusion.}

Obviously the scenario $\underline{I \text { (first) }}$ is optimal. A separation of americium in the scenario II allows decreasing a part of problem. But it is possible that some part of americium or curium can be remained in $\mathrm{HLW}$. Then the relation $\sum \mathrm{Am} / \sum(\mathrm{U}+\mathrm{Np}+\mathrm{Pu}+\mathrm{Cm}+\mathrm{Am})$ can be used to estimate the danger posed by the americium remaining in HLW. It is necessary to note that remains of americium in HLW pose much more danger than remains of curium in HLW.

\section{References.}

1. Y.V.Glagolenko, E.G.Dzekun, C.I.Povniy at. el. Voproci radiacionnoy besopacnocti (Questions of radiation safety). 1997, N 2, p. 3.

2. D.M.Waren, C.Poinssot, P.Baron, M.-C.Charbonnel. $19^{\text {th }}$ International Solvent Extraction Conference ISEC 2011. 3-7 October 2011, Santiago, Chile. P. 105.

3. A.G.Zelenkov, S.V.Pirogikov, V.A.Pcelin at el. Atomic Energy (Russ.). 1981, v. 51, p. 53.

4. N.S.Babaev, A.O.Merkushkin, A.V.Ochkin, C.I.Rovniy. Atomic Energy (Russ.). 1905, v. 98, ed. 2, p. 123. 